\title{
Artificial Intelligence (AI) in Agriculture
}

\author{
V. Dharmaraj* and C. Vijayanand \\ Department of Agriculture Engineering, Sethu Institute of Technology, \\ Viruthunagar 626115, Tamil Nadu, India
}

*Corresponding author

\section{A B S T R A C T}

\begin{tabular}{|l|}
\hline Ke y w o r d s \\
Agriculture, \\
Artificial \\
Intelligence, \\
Robotics, Crop, \\
Farming \\
\hline Article Info \\
\hline $\begin{array}{l}\text { Accepted: } \\
\text { 15 November } 2018 \\
\text { Available Online: } \\
\text { 10 December } 2018\end{array}$ \\
\hline
\end{tabular}

\section{Introduction}

In the $19^{\text {th }}$ century in the times of industrial revolution machines were deployed as a substitution or reduction for human labour. This in course of time, with the advancements and in information technology in the $20^{\text {th }}$ century, post the arrival of the computers, initiated the vision for artificial intelligence (AI) powered machines. In the preset day it's a reality that AI is tardily taking over the human labour.

\section{Scope}

In agriculture there is a quick adaptation to AI in its various farming techniques. The concept of cognitive computing is the one which imitates human thought process as a model in computer. This results as turbulent technology in AI powered agriculture, rendering its service in interpreting, acquiring and reacting to different situations (based on the learning acquired) to enhance efficiency. To harvest benefits in the field by catching up with the recent advancements in farming sector, the farmers can be offered solutions via platforms like chatterbot.

At present in India, Microsoft Corporation is working in the state of Andhra Pradesh with 175 farmers rendering services and solutions for land preparation, sowing, addition of fertilizers and other nutrient supplements for 
crop. On an average, a 30\% increase in crop yield per ha has already been witnessed in comparison to the previous harvests. The various areas where the solutions for benefitting agriculture involving cognition possess knowledge are furnished below.

\section{The Internet of things (IoT) driven development}

There are massive volumes of data getting generated each day in structured and unstructured format. These data are regarding weather pattern, soil reports, new research, rainfall, vulnerability to pest attack, imaging through drones and cameras. IoT solutions relating to cognition would sense, recognize and yield smart solutions to enhance crop yields.

There are two primary technologies deployed for intelligent data fusion, namely proximity and remote sensing. The important application of these high resolution data is for testing the soil. Unlike remote sensing, proximity sensing doesn't need sensors to be built into aerial or satellite systems; it only requires sensors that are in contact with the soil at a close range. This facilitates in the characterization of the soil based on the soil beneath the surface at a particular region.

The hardware solutions like Rowbot (concerning to crops like corn) has already begun pairing software that collect data with robotics to develop the best fertilizer for the cultivation of corns in to maximizing the most possible crop yield.

\section{Image-based insight generation}

In the current world scenario one of the most dissertated areas in farming today is Precision farming. Imaging through drones can assist in rigorous field analysis, in monitoring crops and scanning of fields. With a combination of
Computer vision technology, drone data and IoT will ascertain that the farmers take rapid actions.

Data fed from drone image could bring forth alerts in real time which would accelerate precision farming. Commercial drones makers like Aerialtronics have enforced IBM Watson IoT Platform and the Visual Recognition APIs for eal time image analysis. Some areas computer vision technology can be put to use are as follows,

\section{Disease detection}

The image sensing and analysis ensure that the plant leaf images are sectioned into surface areas like background, diseased area and non diseased area of the leaf. The infected or diseased area is then cropped and sent to the laboratory for further diagnosis. This further renders assistance in the identification of pest and sensing nutrient deficiency. A detailed sequence is presented in figure 1 .

\section{Identify the readiness of the crop}

Images of various crops captured under white light and UVA light are to check how ripe the green fruits are. From this analysis the farmers could create different levels on the readiness of the fruit or crop category. Then add them into assorted stacks before sending them to the market.

\section{Field management}

Employing images of high definition from drone and copters systems, real time estimations can be attained during the time span of cultivation by building a field map and discovering areas where the crops require water, fertilizer and pesticides.

The optimization of resource is assisted to a huge extent by this. 
Identification of optimal mix for agronomic products

Cognitive solutions recommend the farmers on the best choice of crops and hybrid seeds which is grounded on multiple parameters like soil condition, weather forecast, type of seeds and pest infestation in a specific area. A personalized recommendation based on the farm's requirement, native conditions, and data pertaining to successful farming in the past. The other external factors like trends in marketplace, crop prices, consumer needs, requirements and aesthetics may also be factored to enable farmers take a clued-up decision.

\section{Crop health monitoring}

Remote sensing (RS) techniques along with hyper spectral imaging and 3D laser scanning are crucial to construct crop metrics over thousands of acres of cultivable land. It has the potential to introduce a revolutionary shift in how farmlands are monitored by farmers from the perspectives of both time and effort.

This technology will also be employed in monitoring crops throughout their lifecycle including genesis of report in case of abnormalities.

Automation techniques in irrigation and enabling farmers

Irrigation is one of the most labour intensive processes in farming. AI trained machines aware of historical weather pattern, soil quality and kind of crops to be grown, can automate irrigation and increase overall yield.

Nearly $70 \%$ of the world's fresh water resource is utilized for irrigation; such automation can conserve water and benefit farmers in managing their water probs.

\section{Significant of drone}

According to a recent PWC (Price Water House Coopers) study, the total available market for dronebased solutions throughout the world is $\$ 127.3$ billion. And for agriculture is at $\$ 32.4$ billion. Such Drone based solutions in agriculture sector have a lot of implication like dealing with adverse climatic conditions, productivity gains, precision farming and crop yield management.

Fig.1 Disease detection

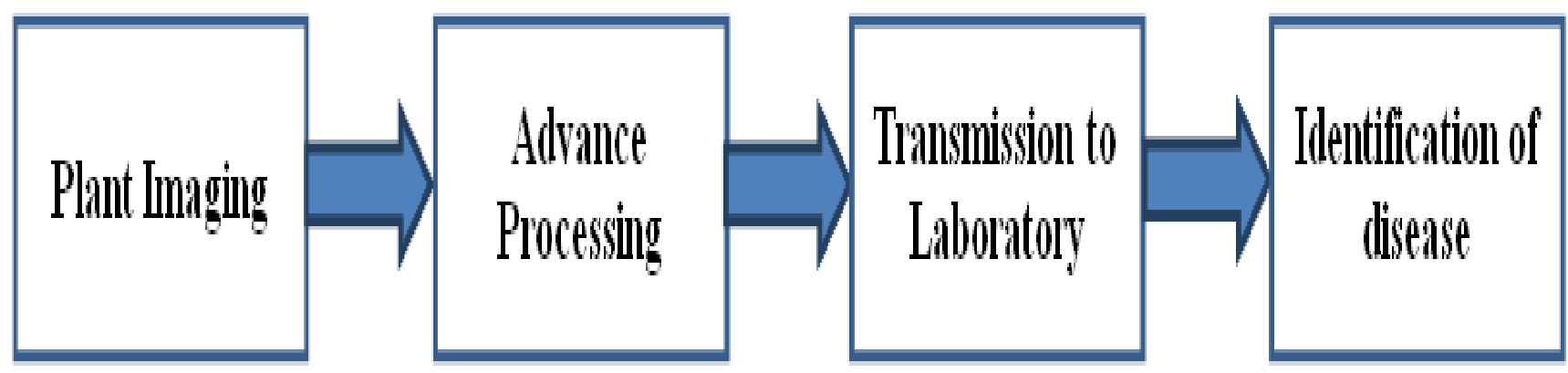


Fig.2 Plant Stress recognition using machine learning and intelligence

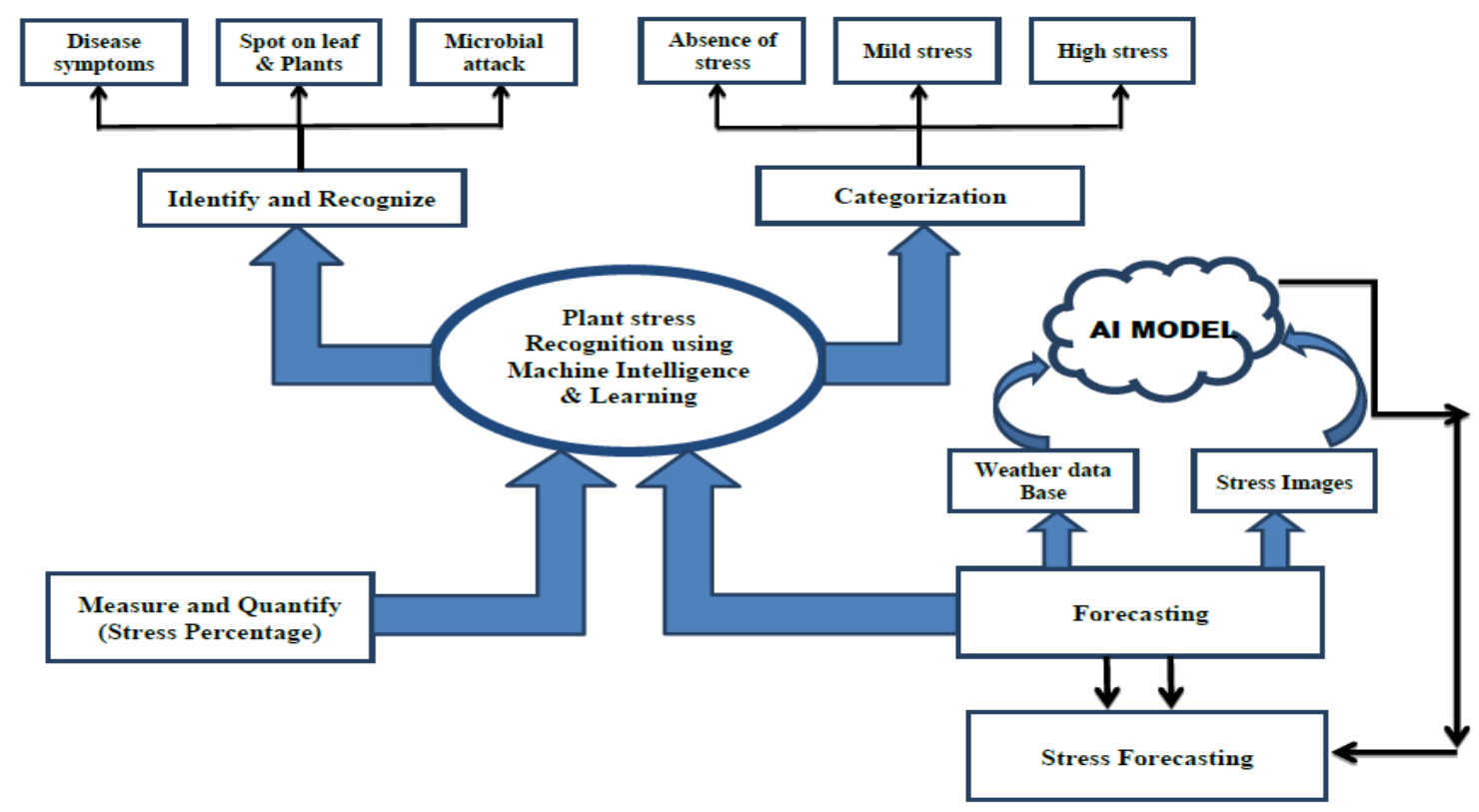

Fig.3 Robotics in digital farming

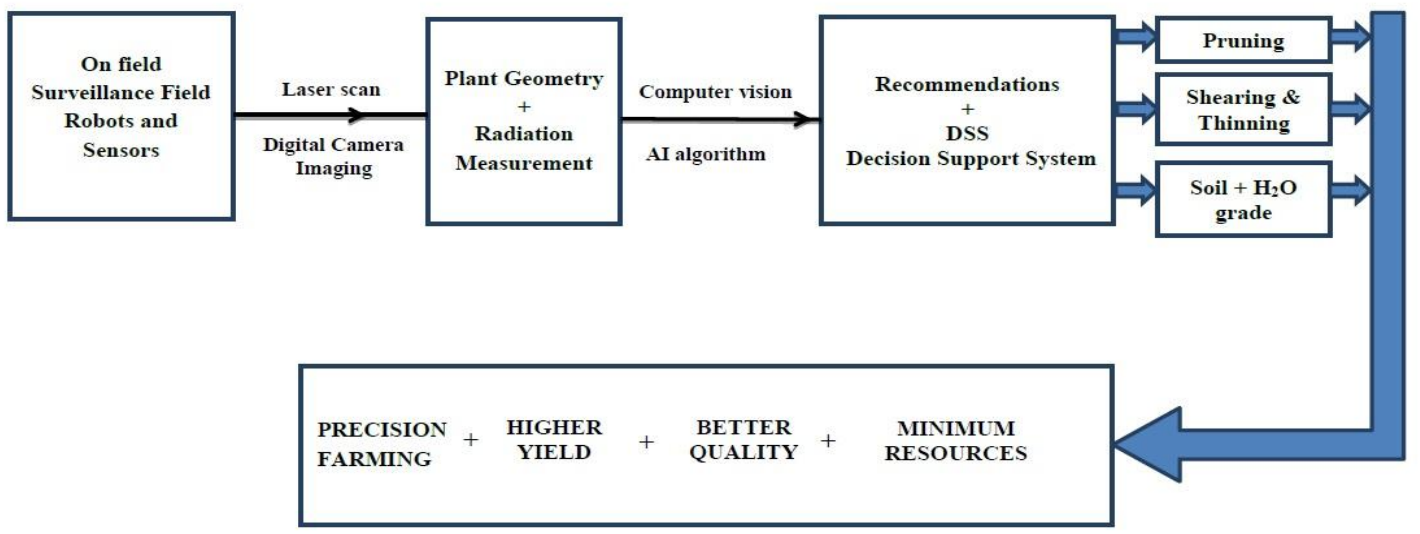

A detailed 3D map of the field, its terrain, irrigation drainage and soil viability must be developed using the drone. This has to be carried out before the crop cycle begins.

The soil $\mathrm{N}_{2}$ levels management can also be done by solutions powered by drone. Drone powered aerial spraying of pods with seeds and plant nutrients into the soil supplies necessary supplements for plants, also the drones can be programmed to atomize liquids by regulating the distance from the ground surface depending on the terrain.

Crop monitoring and crop health assessment prevails as one of the most important domains in agriculture to offer dronebased solutions in coactions with computer vision technology and AI.

Drones with high resolution cameras gather precision field images which can flow 
through convolution neural network to detect areas with weeds, individual crops requiring more water, plant stress level in various growth stages.

In case of infected plants, by scanning crops in both RGB (Red Green Blue) and infra red light, potential multispectral images can be generated using drone devices. Through this individual and specific cluster of plants infected in any region of the field can be spotted and supplied with remedies at once.

The multi spectral images taken from the drone cameras blend hyper spectral images with 3D scanning techniques to define the spatial information system employed for acres of farm land. This renders guidance throughout the lifecycle of the plant as a temporal component.

\section{Precision farming}

Precision farming is a more accurate and controlled technique of farming which substitutes the repetitive and labour intensive part of farming, besides providing guidance regarding crop rotation.

This distinguished key technologies that enable precision farming are high precision positioning system, geological mapping, remote sensing, integrated electronic communication, variable rate technology, optimum planting and harvesting time estimator, water resource management, plant and soil nutrient management, attacks by pest and rodents.

\section{Goals for precision farming}

\section{Profitability}

Recognize crops and market strategically as well as prefiguring ROI (Return on Investment) based on cost and gross profit.

\section{Efficiency}

By putting in precision algorithm, improved, rapid and low cost farming opportunities can be utilized. This lets the overall use of resource efficiently.

\section{Sustainability}

Better socio-economic and environmental operation assures additive improvements in each season for all the performance indicators.

\section{Cases of precision farming management}

The detection of different levels of stress in a plant via high resolution images and multiple sensor data by AI. This entire set of data generated from multiple sources needs to be utilized as an input data for AI machine learning. This enables fusion of these data and features identification parameters for plant stress recognition (Figure 2).

AI machine learning models developed are trained on a wide range of plant images and could recognize the different levels of stress in plants. This total approach can be categorized into four sequential stages of recognition, categorization, quantification and forecasting to take better and improved decisions (Figure 2).

\section{Yield management using AI}

With the emergence of futuristic techs like Artificial Intelligence (AI), cloud machine learning (ML), satellite imaging and advanced analytics are developing an ecosystem for smart, efficient and sustainable farming. The Fusion of these technologies is enabling farmers to achieve higher average yield per ha and better control over the price of food grains, ensuring they remain in profit. 
At present in India, in the state of Andhra Pradesh, Microsoft Corporation is working with farmers rendering farm advisory services using Cortana Intelligence Suite including Machine Learning and Power BI, it enables in transforming the data into Intelligent Actions.

This pilot project makes use of an AI based sowing application which recommends sowing date, preparation of cultivable land, fertigation based on soil analysis, FYM requirement and application, seed treatment and selection, optimization of sowing depth suggestions to the farmers which had resulted in an 30\% increase in the average crop yield per ha.

AI models can also be employed in recognizing optimal sowing period in various seasons, statistical climatic data, real time Moisture Adequacy Data (MAI)from daily rainfall statistics and soil moisture to construct forecast charts and also carter inputs on best sowing time to farmers.

Forecasting potential pest attacks, Microsoft in collaboration with United Phosphorus Limited is developing a Pest Risk Prediction Application Programming Interface (API) that has a strategic advantage of AI and machine learning to signal in advance, the potential chances of pest attack (Figure 3). Grounded on the weather conditions, growth stage of the crop in field, pest attacks are forecast as high, medium or low.

\section{Challenges in AI adoption in agriculture}

Although AI presents immense opportunities in agriculture application, there still prevails a deficiency in familiarity with advanced high tech machine learning solutions in farms around the world. Exposing farming to external factors like weather conditions, soil conditions and vulnerability to the attack of pests is high. A crop raising plan scheduled at the start of the season might not seem to be good at the start of harvesting as it gets influenced by external parameters.

AI systems too require a lot of data for training machines, to take precise forecasting or predictions. Just in case of a very large area of agricultural land, spatial data could be collected easily while getting temporal data is a challenge.

The various crop specific data could be obtained only once in a year when the crops are grown. As the database takes time to mature, it involves a substantial amount of time to construct a robust AI machine learning model. This is a major reason for the utilization of AI in agronomic products like seeds, fertilizer and pesticides than that of on field precision solutions.

In conclusion the future of farming in the times to come is largely reliant on adapting cognitive solutions. Though a vast research is still on and many applications are already available, the farming industry is still not having sufficient service, remains to be underserved. While it comes down in dealing with realistic challenges and demands faced by the farmers, using AI decision making systems and predictive solutions in solving them, farming with $\mathrm{AI}$ is only in a nascent stage.

To exploit the tremendous scope of AI in agriculture, applications should be more robust. Then alone it will be in a position to handle frequent shifts and changes in external conditions. This would facilitate real time decision making and sequentially utilize appropriate model/program for gathering contextual data efficiently.

The other crucial aspect is the extortionate cost of the various cognitive solutions for farming readily available in the market. The 
AI solutions have to become more viable to assure that this technology reaches the farming community. If the AI cognitive solutions are offered in an open source platform that would make the solutions more affordable, which eventually will result in faster adoption and greater insight among the farmers.

\section{References}

Badia Melis. R et al., 2016. "Artificial neural networks and thermal image for temperature prediction in apples," Food and Bioprocess Technology, vol. 9 no.7, pp. 1089-1099.

Balleda, K et al., 2014. "Agpest: An efficient rule-based expert system to prevent pest diseases of rice \& wheat crops," in Proc. Intelligent Systems and Control (ISCO)-2014, IEEE.

Capizzi. G et al., 2016. "A Novel Neural Networks-Based Texture Image Processing Algorithm for Orange Defects Classification," International Journal of Computer Science \& Applications, vol. 13 no. 2, pp. 45-60.

Clark.F.2003. Striking Hypothesis, Human Sci. \& Tech. Press, Changsha.

Hanson A. M. G. J., Joy. A, Francis. J. Plant Leaf Disease Detection using Deep Learning and Convolutional Neural Network, International Journal of Engineering Science, vol. 7 no. 3, pp. 5324-5328, 2017.

Hopfield. J. J. 1982. Neural Networks and Physical Systems with Emergent Collective Computational Abilities, In: Proceedings of the National Academy of Science of the United
States of America, Vol. 79:25542558.

Karmokar B. C., et al., 2015. "Tea leaf diseases recognition using neural network ensemble," International Journal of Computer Applications, vol. 114 no. 17, pp. 27-30.

Polya.G.2004. How to Solve It: A New Aspect of Mathematical Method, Princeton University Press, New Jersey.

Rich. E and Kevin Knight.1991. "Artificial intelligence", New Delhi: McGrawHill.

S. Russell and P. Norvig, 2003. Artificial Intelligence: A Modern Approach, Prentice Hall, New York.

S. Sladojevic, et al., 2016. "Deep neural networks based recognition of plant diseases by leaf image classification," Computational intelligence and neuroscience.

Sharma S.K., K. R. Singh, A. Singh.2010. "An Expert System for diagnosis of diseases in Rice Plant." International Journal of Artificial Intelligence, vol.1 no.1, pp. 26-31.

Taki, M et al., 2016. Application of Neural Networks and multiple regression models in greenhouse climate estimation, Agricultural Engineering International: CIGR Journal, vol. 18 no. 3 , pp. 29-43.

Xingsan Hu. 1996. Tracing back to the history of AI, Journal of Lishui Normal School, Vol. 18, No. 5: 20-22.

Zixing Cai and Guangyou Xu, 2004. Artificial Intelligence and Its Applications, The $3^{\text {rd }}$ Edition, Tsinghua University Press, Beijing.

\section{How to cite this article:}

Dharmaraj, V. and Vijayanand, C. 2018. Artificial Intelligence (AI) in Agriculture. Int.J.Curr.Microbiol.App.Sci. 7(12): 2122-2128. doi: https://doi.org/10.20546/ijcmas.2018.712.241 